\title{
Transtornos de ansiedade em usuários de substâncias de um Centro de Atenção Psicossocial álcool e drogas
}

\author{
Anxiety disorders in substance users at analcohol and drugs Psychosocial Care Center \\ Trastornos de ansiedad en usuários de sustâncias en un Centro de Atención Psicosocial \\ alcohol y drogas
}

Selva Rios Campêlo ${ }^{1 \star}$, Maria Alves Barbosa ${ }^{1}$, Danilo Rocha Dias².

\section{RESUMO}

Objetivo: Identificar a prevalência de transtornos de ansiedade dos usuários de substâncias de um Centro de Atenção Psicossocial álcool e drogas (CAPSad), com ênfase na severidade da dependência. Métodos: Estudo transversal com 60 usuários de em um CAPSad. Foi utilizado o Mini International Neuropsychiatric Interview (MINI) para avaliar a prevalência de transtornos de ansiedade e a escala Addiction Severity Index (ASI) para avaliar a severidade da dependência. Foi realizada estatística descritiva e testes de comparação $(p<0,05)$. O estudo foi aprovado pelo Comitê de Ética em Pesquisa. Resultados: Os participantes eram na maioria homens, com idade média de 38 anos. A maior parte deles apontou a cocaína/crack como a principal substância que os motivou a buscar o tratamento. A prevalência de transtornos de ansiedade atual foi de $50 \%$, sendo os transtornos de pânico e de ansiedade generalizada os mais prevalentes com $31,7 \%$ e $28,3 \%$ respectivamente. Os participantes com transtornos de ansiedade apresentaram maior severidade da dependência principalmente nos domínios psiquiátrico, álcool e problemas sociofamiliares. Conclusão: A prevalência de transtornos de ansiedade é alta em usuários de substâncias em tratamento e está relacionada com maior severidade da dependência. São necessários estudos que possibilitem uma análise mais detalhada desse tipo de comorbidade.

Palavras-chave: Transtornos relacionados ao uso de substâncias, Transtornos de ansiedade, Prevalência.

\begin{abstract}
Objective: To identify the prevalence of anxiety disorders among substance users at a Psychosocial Care Center for alcohol and drugs (CAPSad), with an emphasis on the severity of dependence. Methods: Cross sectional study with 60 users of a CAPSad. The Mini International Neuropsychiatric Interview (MINI) was used to assess the prevalence of anxiety disorders and the Addiction Severity Index (ASI) scale to assess the severity of addiction. Descriptive statistics and comparison tests were performed $(p<0.05)$. The study was approved by the Research Ethics Committee. Results: The participants were mostly men, with an average age of 38 years. Most of them identified cocaine/crack as the main substance that motivated them to seek treatment. The prevalence of current anxiety disorders was $50 \%$, with panic and generalized anxiety disorders being the most prevalent with $31.7 \%$ and $28.3 \%$ respectively. Participants with anxiety disorders showed greater severity of dependency, especially in the psychiatric, alcohol and family social problems. Conclusion: The prevalence of anxiety disorders is high in users of substances being treated and is related to greater severity of dependence. Studies are needed to enable a more detailed analysis of this type of comorbidity.
\end{abstract}

Keywords: Substance-related disorders, Anxiety disorders, Prevalence.

1 Universidade Federal de Goiás (UFG), Goiânia - GO.

2 Universidade Federal de Minas Gerais (UFMG), Belo Horizonte - MG.

^E-mail: selvariosps@hotmail.com.

SUBMETIDO EM: $8 / 2020$

ACEITO EM: 9/2020

PUBLICADO EM: $11 / 2020$

REAS/EJCH | Vol.12(11) | e4917 | DOI: https://doi.org/10.25248/reas.e4917.2020 Página 1 de 9 


\section{RESUMEN}

Objetivo: Identificar la prevalencia de los trastornos de ansiedad entre los usuarios de sustancias en un Centro de Atención Psicosocial para el alcohol y las drogas (CAPSad), con énfasis en la severidad de la dependencia. Métodos: Estudio transversal con 60 usuarios de un CAPSad. El Mini International Neuropsychiatric Interview (MINI) se utilizó para evaluar la prevalencia de los trastornos de ansiedad y la escala del Índice de Severidad de la Adicción (ASI) para evaluar la severidad de la adicción. Se realizaron estadísticas descriptivas y pruebas de comparación $(p<0.05)$. El estudio fue aprobado por el Comité de Ética en Investigación. Resultados: Los participantes eran en su mayoría hombres, con una edad media de 38 años. La mayoría de ellos identificaron la cocaína/crack como la sustancia principal que los motivó a buscar tratamiento. La prevalencia de los trastornos de ansiedad actuales fue del $50 \%$, siendo los trastornos de pánico y ansiedad generalizada los más prevalentes con $31.7 \%$ y $28.3 \%$ respectivamente. Los participantes con trastornos de ansiedad mostraron una mayor severidad de dependencia, especialmente en los problemas psiquiátricos, alcohólicos y sociales familiares. Conclusión: La prevalencia de los trastornos de ansiedad es alta en los usuarios de sustancias que están siendo tratadas y está relacionada con una mayor severidad de la dependencia. Se necesitan estudios para permitir un análisis más detallado de este tipo de comorbilidad.

Palabras clave: Trastornos relacionados con uso de sustancias, Trastornos de ansiedad, Prevalencia.

\section{INTRODUÇÃO}

Resultados do estudo Global Burden of Disease 2016 (GDB) mostram que 2,22\% da população mundial e 3,73\% da população brasileira tem um transtorno por uso de álcool ou drogas (), que é caracterizado pela presença de um grupo de sintomas cognitivos, comportamentais e fisiológicos devido ao uso continuado de uma ou mais substâncias a despeito dos problemas significantes gerados pelo seu uso (GDB, 2016; APA, 2014; RITCHIE H, 2019). Em seus graus mais severos, o transtorno é marcado pela cronicidade e recorrência, representando um grande desafio para os sistemas de saúde em todas as regiões do mundo (TRAN BX, et al., 2019; KOOB GF e VOLKOM ND, 2010).

Transtornos de ansiedade e transtornos por uso de substâncias têm sido reportado como altamente prevalentes e fortemente associados (REGIER DA, et al., 1990, MERIKANGAS KR, et al., 1998, KESSLER RC, et al., 2005, GRANT BF, et al., 2004, LAI HM, et al., 2015). A comorbidade entre o transtorno de ansiedade e o transtorno por uso de substâncias afeta ambos os transtornos no perfil, curso, padrões, gravidade e resultados de tratamento (LAI HM, et al., 2015, SMITH JP e BOOK SW, 2008).

Avanços na neurociência e na medicina do comportamento mostram que os problemas mentais e comportamentais resultam de uma complexa interação de fatores biológicos, psicológicos e sociais (WHO, 2001).

A presença de transtornos mentais nos usuários de substâncias está associada a uma pior qualidade de vida e pior funcionamento psicossocial com mais problemas na área ocupacional, na saúde física e mental, nos relacionamentos sociais e familiares, aumentando a dificuldade nos tratamentos (TORRENS M, et al., 2015; KESSLER F, et al., 2010). Transtornos de ansiedade além de ter uma alta prevalência entre os usuários de substâncias está associado a uma série de maus resultados psicossociais, incluindo taxas mais altas de hospitalização, incapacidade, comprometimento funcional e resultados inferiores do tratamento (MAGIDSON JF, et al., 2012).

Contudo, vale ressaltar que a forma como os problemas pelo uso de substâncias afetam ou se relacionam com a vida do usuário pode ser diferente para os indivíduos. Por exemplo, enquanto alguns apresentam mais problemas na área social, outros apresentam maiores problemas na área psicológica ou de saúde física.

Identificar a prevalência de transtornos de ansiedade em usuários de substâncias em tratamento é fundamental para a identificação de prioridades e intervenções necessárias e pode contribuir assim para um tratamento mais efetivo para os transtornos por uso de substâncias, com melhor entendimento diagnóstico e melhores possibilidades terapêuticas. 
Este artigo tem como objetivo identificar a prevalência de transtornos de ansiedade dos usuários de substância atendidos em um Centro de Atenção Psicossocial álcool e drogas (CAPSad), com ênfase na severidade da dependência.

\section{MÉTODOS}

Este estudo transversal está aninhado a uma pesquisa mais ampla, que avaliou a correlação entre severidade da dependência de drogas e a qualidade de vida de 60 usuários em tratamento em um CAPSad (CAMPÊLO SR, et al., 2017). Nesta pesquisa, observou-se uma alta prevalência de transtornos de ansiedade entre os participantes, com uma diferença significativa no domínio psicológico da qualidade de vida entre os que tinham e os que não tinham este transtorno. Estes resultados mostraram a necessidade de uma análise mais detalhada de transtornos de ansiedade nessa população, considerando-se os diferentes tipos de transtornos de ansiedade, com foco nos transtornos atuais.

O tratamento para transtornos por uso de substâncias nesse centro de atenção é oferecido pelo Sistema Único de Saúde (SUS) à população adulta, visando o tratamento médico, psiquiátrico e a recuperação psicossocial dos usuários. A amostra incluiu todos os indivíduos com uso de drogas, podendo ter o uso de álcool associado, que concordaram em participar e assinaram o Termo de Consentimento livre e Esclarecido. Aqueles com dificuldade de compreensão cognitiva no momento da entrevista foram excluídos. O processo de amostragem encontra-se descrito detalhadamente em publicação anterior (CAMPÊLO SR, et al., 2017).

Foram utilizadas as versões validadas para a cultura brasileira dos seguintes instrumentos: o Mini International Neuropsychiatric Interview (MINI) versão 5.0, para identificar a presença de transtornos de ansiedade de acordo com o DSM-IV, e a escala Addiction Severity Index (ASI-6) para colher dados sociodemográficos e de severidade da dependência (AMORIM P, 2000; KESSLER F, et al., 2012). A coleta de dados foi feita individualmente e a aplicação dos instrumentos foi realizada pela pesquisadora responsável (SRC), e por assistentes de pesquisa treinadas e supervisionadas.

O MINI é um instrumento de entrevista para avaliação diagnóstica padronizada dos transtornos mentais. Os critérios diagnósticos da versão 5.0 são compatíveis com os critérios diagnósticos do DSM-IV (APA, 2008). Foram utilizados para esta pesquisa os módulos que pesquisam os transtornos atuais de pânico, com ou sem agorafobia, fobia social, transtorno obsessivo compulsivo, transtornos de estresse pós-traumático e transtorno de ansiedade generalizada.

A ASI é uma escala que tem como objetivo avaliar a severidade da dependência dos usuários de substâncias nos vários domínios de sua vida. A escala avalia sete dimensões: médica, empregos e finanças, legal, psiquiátrica, álcool, drogas e família/social. Um escore é calculado para cada dimensão com exceção da dimensão família/social que calcula três escores: problemas sociofamiliares, apoio sociofamiliar e família/crianças.

Para compreensão dos dados colhidos foi realizada estatística descritiva e inferencial utilizando-se o programa estatístico IBM-SPSS - Statistical Program of Social Science versão 23,0. A amostra foi dividida em grupos definidos pela presença ou não dos transtornos de ansiedade, que foram comparados utilizando teste t de Student e teste de Mann-Whitney, de acordo com a normalidade de distribuição dos dados, verificada pelo teste de Shapiro-Wilk. Para todas as análises foi considerado o nível de significância em $95 \%$.

Este projeto segue diretrizes éticas brasileiras, regulamentadas pela resolução do Conselho Nacional de Saúde 466/2012, tendo sido aprovado pelo Comitê de Ética em Pesquisa (CEP) da Universidade Federal de Goiás, parecer número 927256, CAAE 37419814.5.0000.5078.

\section{RESULTADOS}

A amostra incluiu 60 participantes, dos quais 55 eram homens com a idade média de 38 anos (19-59). A maior parte dos participantes apontou cocaína/crack como a substância que mais os motivou a buscar tratamento (Tabela 1). 
Tabela 1 - Substâncias que motivaram os usuários a buscarem tratamento, hierarquizadas de acordo com a motivação pela busca de tratamento, CAPSad, 2020.

\begin{tabular}{cccc}
\hline Substâncias & $\begin{array}{c}\text { Problema principal } \\
\mathbf{n}(\%)\end{array}$ & $\begin{array}{c}\text { 20 Problema } \\
\mathbf{n}(\%)\end{array}$ & $\begin{array}{c}\mathbf{3}^{\circ} \text { Problema } \\
\mathbf{n}(\%)\end{array}$ \\
\hline Cocaína/Crack & $32(53,3)$ & $15(25)$ & $4(6,7)$ \\
\hline Álcool & $15(25)$ & $15(25)$ & $2(3,3)$ \\
\hline Maconha & $11(18,3)$ & $12(20)$ & $4(6,7)$ \\
\hline Outras substâncias & $2(3,3)$ & 0 & 0 \\
\hline
\end{tabular}

Fonte: Campêlo SR, et al., 2020.

A prevalência total dos transtornos do espectro de ansiedade atual (qualquer transtorno de ansiedade atual) foi de $50 \%$. Os mais prevalentes foram o transtorno de pânico com $31,7 \%$ e o transtorno de ansiedade generalizada com $28,3 \%$ (Tabela 2 ).

Tabela 2 - Frequência de transtornos do espectro de ansiedade atual, CAPSad, 2020.

\begin{tabular}{cc}
\hline Transtornos & $\mathbf{n}(\%)$ \\
\hline Qualquer transtorno de ansiedade atual & $30(50,0)$ \\
Transtorno de pânico (com/sem agorafobia) & $19(31,7)$ \\
Transtorno de ansiedade generalizada & $17(28,3)$ \\
Transtorno obsessivo compulsivo & $14(23,3)$ \\
Transtorno de estresse pós-traumático & $12(20,0)$ \\
Fobia social & $11(18,3)$
\end{tabular}

Fonte: Campêlo SR, et al., 2020

Os dados coletados pela escala ASI revelaram a severidade da dependência em nove domínios. Os valores médios para cada domínio foram: 49,45 para drogas, 50,10 para álcool, 49,92 para psiquiátrico, 48,18 para médico, 48,68 para legal, 41,63 para emprego 51,88 para família/criança, 49,68 para apoio sócio familiar e 52,55 para problema sócio familiar conforme relatado em pesquisa anterior (CAMPÊLO SR, et al., 2017).

O grupo de participantes com qualquer transtorno de ansiedade mostrou pior severidade na ASI, com diferença estatisticamente significativa nos domínios álcool, psiquiátrico, médico e problemas sócios familiares (Tabela 3).

Considerando cada transtorno de ansiedade separadamente, todos os transtornos de ansiedade mostraram diferenças significativas com pior severidade da dependência para os participantes com algum transtorno de ansiedade em pelo menos um domínio da escala ASI, sendo os domínios psiquiátricos, álcool e problemas sociofamiliares os mais presentes. A severidade da dependência no domínio psiquiátrico foi maior para todos os transtornos com exceção do transtorno de ansiedade generalizada. A severidade da dependência para o domínio álcool foi maior para os transtornos de pânico, obsessivo compulsivo e estresse pós-traumático. Por último, a severidade da dependência para o domínio problemas sociofamiliares foi maior para os transtornos de ansiedade generalizada e estresse pós-traumático (Tabela 3). 


\section{Revista Eletrônica Acervo Saúde / Electronic Journal Collection Health | ISSN 2178-2091}

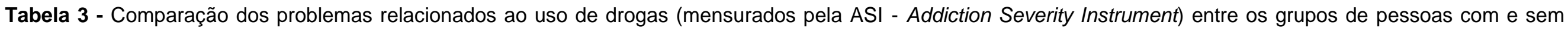
transtornos do espectro de ansiedade atual, CAPSad, 2020.

\section{Tipos de problemas de acordo com os domínios da ASI}

\begin{tabular}{|c|c|c|c|c|c|c|c|c|c|}
\hline Comorbidades & Drogas & Família/ crianças & Álcool & Psiquiátrico & Médico & Legal & Emprego & ASF & PSF \\
\hline & (Media/DP) & (Media/DP) & (Media/DP) & (Media/DP) & (Media/DP) & (Media/DP) & (Media/DP) & (Media/DP) & (Media/DP) \\
\hline \multicolumn{10}{|c|}{ Qualquer TA } \\
\hline Sim & $50,8(6,8)$ & $53,2(8,3)$ & $54(9,2)$ & $54,8(7)$ & $50,5(8,3)$ & $49,4(5,2)$ & $41,4(10,7)$ & $49,9(10)$ & $56(9,9)$ \\
\hline Não & $48,4(6,7)$ & $49,4(4,2)$ & $46,8(8,6)$ & $45,4(5,5)$ & $45,8(8,8)$ & $48,1(5,1)$ & $42,1(11,7)$ & $49(10,8)$ & $49,1(6)$ \\
\hline Valor de $\mathrm{p}$ & $0,08^{\mathrm{b}}$ & $0,052^{b}$ & $0,004^{* \mathrm{~b}}$ & $0,000 * a$ & $0,03^{* a}$ & $0,22^{\mathrm{b}}$ & $0,99^{b}$ & $0,58^{a}$ & $0,002^{* a}$ \\
\hline \multicolumn{10}{|c|}{ TP com/sem agorafobia } \\
\hline Sim & $50,4(5,8)$ & $52(7,2)$ & $56,3(5,6)$ & $55,2(7,6)$ & $50,9(10)$ & $49,6(5,5)$ & $43,2(10,1)$ & $52,4(11,2)$ & $54(11,8)$ \\
\hline Valor de $p$ & $0,48^{\mathrm{a}}$ & $0,83^{\mathrm{b}}$ & $0,000^{* b}$ & $0,00 * a$ & $0,1^{\mathrm{a}}$ & $0,26^{b}$ & $0,49^{b}$ & $0,17^{a}$ & $0,49^{a}$ \\
\hline \multicolumn{10}{|c|}{ TAG } \\
\hline Sim & $51,5(7,2)$ & $57,4(9,6)$ & $51,4(10,4)$ & $51,8(6,1)$ & $49,3(6,8)$ & $47,2(3,5)$ & $41,1(11,6)$ & $48,1(10,3)$ & $58,4(9,9)$ \\
\hline Não & $48,7(6,6)$ & $49,7(4,9)$ & $49,6(9,2)$ & $49,2(8,3)$ & $47,7(9,3)$ & $49,3(5,6)$ & $41,8(11,3)$ & $50,3(10,4)$ & $50,3(7,4)$ \\
\hline Valor de $p$ & $0,09^{\mathrm{b}}$ & 0,00 *b & $0,66^{\mathrm{b}}$ & $0,25^{\mathrm{a}}$ & $0,54^{\mathrm{a}}$ & $0,18^{\mathrm{b}}$ & $0,84^{b}$ & $0,47^{a}$ & $0,001^{* a}$ \\
\hline \multicolumn{10}{|c|}{ TOC } \\
\hline Sim & $51,1(5,3)$ & $51,1(8)$ & $55,6(8,5)$ & $55,6(6,6)$ & $49,6(7,5)$ & $51,6(5,9)$ & $42,9(10,2)$ & $54,1(9,5)$ & $56,6(10,3)$ \\
\hline Não & $48,9(7,2)$ & $52,1(7,2)$ & $48,4(9,3)$ & $48,2(7,4)$ & $47,8(9)$ & $47,8(4,6)$ & $41,3(11,6)$ & $48,4(10,3)$ & $51,4(8,1)$ \\
\hline Valor de $p$ & $0,29^{a}$ & $0,52^{\mathrm{b}}$ & $0,01^{* \mathrm{~b}}$ & $0,001^{* a}$ & $0,5^{\mathrm{a}}$ & $0,01^{* b}$ & $0,66^{\mathrm{b}}$ & $0,07^{a}$ & $0,06^{\mathrm{a}}$ \\
\hline Sim & $51,8(6,2)$ & $53,5(8,5)$ & $55,5(7,4)$ & $57,9(6,4)$ & $51(8,2)$ & $49,6(5,3)$ & $43,1(11,6)$ & $51,9(8,9)$ & $59,7(9,4)$ \\
\hline Não & $48,9(6,9)$ & $51,5(7,1)$ & $48,8(9,6)$ & $47,9(6,8)$ & $47,5(8,7)$ & $48,5(5,1)$ & $41,3(11,3)$ & $49,1(10,7)$ & $50,7(7,8)$ \\
\hline Valor de $p$ & $0,11^{\mathrm{b}}$ & $0,38^{b}$ & 0,01 *b & $0,00 * a$ & $0,21^{a}$ & $0,32^{b}$ & $0,54^{b}$ & $0,41^{a}$ & $0,004^{\star b}$ \\
\hline \multicolumn{10}{|c|}{ FS } \\
\hline Sim & $51,6(6,5)$ & $54,1(8,4)$ & $56(7,3)$ & $55(4,3)$ & $49,4(8,9)$ & $47,1(3,5)$ & $44,6(11,5)$ & $46,4(10)$ & $57,2(10,1)$ \\
\hline Não & $49,2(6,9)$ & $50,7(6,3)$ & $49,2(9,6)$ & $49,1(8,1)$ & $47,9(8,8)$ & $49,1(5,4)$ & $41,2(11,1)$ & $50,1(10,4)$ & $51,6(8,3)$ \\
\hline Valor de $p$ & $0,16^{b}$ & $0,06^{\mathrm{b}}$ & $0,07^{b}$ & $0,049^{* a}$ & $0,54^{a}$ & $0,25^{\mathrm{b}}$ & $0,18^{\mathrm{b}}$ & $0,6^{a}$ & $0,07^{a}$ \\
\hline
\end{tabular}

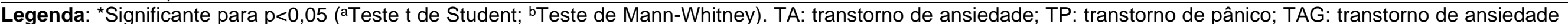
generalizada; TOC: transtorno obsessivo compulsivo; TEPT: transtorno de estresse pós-traumático; FS: fobia social.

Fonte: Campêlo SR, et al., 2020. 


\section{DISCUSSÃO}

O resultado dessa pesquisa mostrou que $50 \%$ dos usuários que estavam em tratamento para o uso de substâncias apresentavam também algum transtorno atual de ansiedade. Os transtornos de ansiedade mais prevalentes foram os transtornos de pânico, com ou sem agorafobia, e o transtorno de ansiedade generalizada, seguidos pelo transtorno obsessivo compulsivo, transtorno de estresse pós-traumático e fobia social.

Esses resultados estão de acordo com pesquisas de bases comunitárias que mostram alta prevalência de comorbidades de transtornos de ansiedade e transtornos por uso de substâncias (REGIER DA, et al., 1990, GRANT BF, et al., 2004).

O Epidemiology Catchement Area - (ECA) relatou que na população geral e institucionalizada a prevalência de transtornos de ansiedade na vida era de $19,4 \%$ para pessoas com transtorno por uso de álcool e de $28,3 \%$ para transtorno por uso de drogas (REGIER DA, et al., 1990).

O National Epidemiologic Survey in Alcohol and Related Conditions (NESARC) relatou, considerando a população geral nos últimos 12 meses, que $17,71 \%$ das pessoas com algum transtorno por uso de substâncias tinham no mínimo um transtorno de ansiedade independente (GRANT BF, et al., 2004). As taxas foram mais altas para dependência de substâncias do que para abuso e para dependência de drogas do que de álcool (GRANT BF, et al., 2004). Entre os respondentes que declararam ter buscado tratamento para o uso de álcool 33.38\% tinham transtorno de ansiedade e 33.05\% tinham transtorno por uso de drogas (GRANT BF, et al., 2004).

Entre os respondentes que buscaram tratamento para o uso de drogas $42,63 \%$ tinham transtorno de ansiedade e 55,16\% tinham transtornos de álcool. As prevalências de transtornos específicos de ansiedade para os que tinham transtorno por uso de álcool foi de 13,20\% para pânico com e sem agorafobia, 12,35\% para transtorno de ansiedade generalizada e $8,49 \%$ para fobia social. Para os que tinham transtornos por uso de drogas foi de $14,56 \%$ para pânico com e sem agorafobia, 22,07\% para transtorno de ansiedade generalizada e 12,09\% para fobia social (GRANT BF, et al., 2004). Neste estudo não estavam incluídos transtorno de estresse pós traumático e transtornos obsessivo compulsivo.

O fato das prevalências encontradas na presente pesquisa serem maiores do que as das pesquisas epidemiológicas citadas pode ser explicado pelo tipo de população, sugerindo que em ambientes de tratamento as prevalências são maiores do que as observadas em estudos de bases comunitárias (REGIER DA, et al., 1990).

Um outro estudo realizado em CAPSad encontrou prevalências de transtornos de ansiedade semelhantes às encontradas neste, com exceção do transtorno de ansiedade generalizada que obteve uma prevalência bem maior, de $63,10 \%$. Os outros transtornos de ansiedade foram $36,89 \%$ para transtorno de estresse póstraumático, 31,06\% para o transtorno de pânico, 22,33\% para o transtorno obsessivo compulsivo e 19,41\% para a fobia social (ANDRADE JDS, 2014).

Outra pesquisa realizada em CAPSad encontrou prevalências menores sendo de 17,9\% para transtorno de ansiedade generalizada, 12,8\% para transtorno de estresse pós-traumático, 10,3\% para fobia social, 7,7\% para transtornos de pânico e 2,6\% para transtorno obsessivo compulsivo (FORMIGA MB, et al., 2015).

Comparados com os resultados aqui descritos percebemos que essas pesquisas mostram uma ordem de maior prevalência diferente. Essas diferenças nas taxas de prevalência e na ordem de maiores prevalências podem ser talvez explicadas por variações clínicas, com características específicas de regionalidade, tipos de substâncias e tempo de tratamento, entre outros aspectos. Nota-se a necessidade de mais estudos de comorbidades de transtornos de ansiedade e transtornos por uso de substâncias em CAPSad.

Além da variabilidade nas taxas de prevalência os transtornos variam quanto ao grau de severidade sendo normalmente mais severos em ambientes de tratamento, e com mais comorbidades psiquiátricas (REGIER DA, et al., 1990; KESSLER RC, et al., 2005). 
A severidade e comprometimento dos diferentes transtornos mentais podem variar substancialmente. Os transtornos de depressão, pânico, agorafobia, fobia social, transtorno de ansiedade generalizada, dependência de álcool e de drogas mostraram ser independentemente associados a comprometimento e incapacitações entre participantes de um estudo em base comunitária, após ajustamento para os dados sociodemográficos e doenças físicas (SANDERSON K e ANDREWS G, 2002).

Neste estudo a severidade dos transtornos por uso de substâncias foi avaliada pela ASI constatando-se pior severidade no grupo de pessoas com transtornos de ansiedade, principalmente nos domínios psiquiátrico, álcool e problemas sócio familiares. Todos os transtornos de ansiedade com exceção do transtorno de ansiedade generalizada apresentaram maior severidade da dependência no domínio psiquiátrico, o qual foi o domínio que mais apresentou diferenças significativas entre todos os transtornos.

$\mathrm{Na}$ ASI a severidade psiquiátrica é avaliada por questões relacionadas a preocupações e necessidade de receber tratamentos psicológicos e psiquiátricos e com problemas psiquiátricos tais como dificuldade de sono, sentir-se deprimido ou ansioso, ter alucinações, dificuldade de concentração, dificuldade para controlar temperamento, impulsividade, ideação ou tentativas de suicídio. Esses sintomas são característicos de diversos transtornos mentais e não somente de transtornos por uso de substâncias o que corrobora com a severidade dos transtornos estar associada a presença de outras comorbidades (APA, 2008).

Além de ser comum a associação de transtornos por uso de substâncias e transtornos de ansiedade os transtornos de ansiedade são comumente associados entre si e com o transtorno de humor, que é o transtorno com maior probabilidade de ter maior número de casos mais severos entre os transtornos mentais (VERONA E, et al., 2004; KRUEGER RF, 1999; KESSLER et al., 1997; KESSLER RC, et al., 2005). Pode-se inferir daí que as pessoas que apresentaram maior severidade psiquiátrica terão provavelmente maior quantidade de comorbidades e maior dificuldade para o tratamento.

A presença de comportamento suicida, tais como ideação ou tentativas de suicídio é um forte indicativo da gravidade psiquiátrica (KESSLER RC, et al., 2005). Dados do Netherlands Mental Health Survey and Incidence Study (NEMESIS) mostraram associação significativa entre os transtornos de ansiedade e comportamento suicida, mesmo após ajustamento para fatores sociodemográficos e presença de outros transtornos mentais (SAREEN J, et al., 2005). Além disso, comorbidade de transtornos de ansiedade ampliam o risco de tentativas de suicídio em pessoas que têm transtornos de humor (SAREEN J, et al., 2005).

O domínio álcool foi o segundo domínio com mais diferenças entre os grupos com a presença ou ausência de transtornos de ansiedade apresentando maior severidade nos transtornos de pânico, obsessivo compulsivo e de estresse pós traumático. O domínio álcool da ASI avalia questões relativas à frequência e problemas decorrentes do uso de álcool além de preocupações ou necessidade de receber tratamentos psicológicos e psiquiátricos para essa área.

Tanto o transtorno por uso de álcool pode desencadear um transtorno de ansiedade quanto o transtorno de ansiedade pode desencadear um transtorno por uso de álcool (KUSHNER MG, et al., 2000). Estudos clínicos sugerem que transtornos de ansiedade contribuem para a manutenção e recaída do álcool (KUSHNER MG, et al., 2000), dificultando o tratamento do transtorno por uso de álcool.

Equação estrutural de variáveis latentes, mostrou que uma grande proporção de covariantes em transtornos de ansiedade e humor podem ser canalizados ao longo de um único continuum denominado "emocionalmente negativo" (KRUEGER RF, 1999). Sendo assim, todos os transtornos de ansiedade e humor contribuem para a "emoção negativa geral" o que por sua vez correlaciona com o risco de dependência de álcool (ANKER JJ e KUSHNER MG, 2019). O que se conclui que a dependência de álcool pode estar mais correlacionada com a quantidade de comorbidades, inclusive de ansiedade, do que com cada transtorno específico de ansiedade.

Diferentemente do domínio álcool, o domínio droga não encontrou diferenças entre os grupos. Isso não seria o esperado visto que as drogas, normalmente acarretam mais problemas devido ao uso do que o álcool e possuem maior associação com os transtornos de ansiedade (MERIKANGAS KR, et al., 1998; LAI HM, et al., 2015). 
Talvez isso possa ser justificado devido ao critério de inclusão dessa presente pesquisa ter sido sujeitos com problemas de drogas, que podiam ter ou não o uso associado de álcool, o que torna difícil a diferenciação entre os problemas da ASI em relação ao uso de drogas e de álcool, pois aqueles que tinham o problema pelo uso de álcool tinham problemas também com drogas, o que pode ter contribuído para a maior severidade na ASI deste domínio.

A maior gravidade de transtornos por uso de substâncias está entre aqueles com poliuso de substâncias sendo o álcool muito frequente entre os usuários de cocaína/ crack, que foi a principal substância declarada como motivo para buscar o tratamento nesta pesquisa (ABP, 2012).

O domínio problemas sociofamiliares foi o terceiro domínio com mais diferenças entre os grupos com a presença ou ausência de transtornos de ansiedade, apresentando maior severidade para os participantes com transtorno de ansiedade generalizada e transtorno de estresse pós traumático.

A severidade na área de problemas sociofamiliares da ASI é avaliada por questões relacionadas a problemas de relacionamentos com parceiros, parentes adultos e amigos íntimos e a preocupações e necessidade de receber tratamentos psicológicos e psiquiátricos para essa área.

O uso abusivo de substâncias normalmente impacta a família do usuário de substâncias, sendo a forma de relacionar com violência umas das piores consequências que a família pode experimentar (KESSLER F, et al., 2010, IRONS R e SCHNEIDER JP, 1997). O álcool pode facilitar a violência agindo como desinibidor e estimulantes como cocaína/crack podem antecipar comportamento violento ao reduzir o controle de impulsos e aumentar sentimentos paranoicos (ZILBERMAN ML e BLUME SB, 2005). Dificuldade em controlar agressão e raiva em pessoas com transtorno de ansiedade é possivelmente associada a consequências negativas, como violências físicas, destruição de propriedades e ameaças de violência (KEYES KM, et al., 2016).

$\mathrm{O}$ aumento na gravidade de sintomas de transtorno de ansiedade generalizada foram associadas com ações impulsivas que é comum nas pessoas com transtornos de ansiedade (PAWLUK EJ e KOERNER N, 2013; JAKUSZKOWIAK-WOJTEN K, et al., 2015).Também pacientes com transtornos de pânico, fobia social e transtorno obsessivo compulsivo relataram níveis mais altos de impulsividade do que população não clínica (SUMMERFELDT LJ, et al., 2004).

A exposição a eventos traumáticos é frequente aos usuários de substâncias e pode levar ao desenvolvimento de transtorno de estresse pós-traumático (SMITH NDL e COTTLER LB, 2018). O nível de estresse dos pais está associado a sintomas de estresse pós-traumáticos nos filhos e provavelmente causa problemas familiares. Isso pode explicar a maior severidade da dependência no domínio problemas sociofamiliares em pessoas com transtorno de estresse pós-traumático, observada neste estudo (BRYANT RA, et al., 2018).

Este presente estudo é limitado devido não ter analisado a presença associada de outros transtornos mentais pois, como já exposto anteriormente, a presença de várias comorbidades é comum na saúde mental e além da possibilidade de alterar a sintomatologia dos transtornos está mais relacionada a severidade do que a especificidade de cada transtorno. São necessários mais estudos sobre a prevalência de transtornos de ansiedade nos CAPSad e outros lugares de tratamento para uso de substâncias e sobre a gravidade dessa comorbidade nos domínios da vida dos usuários.

\section{CONCLUSÃO}

Conclui-se que a prevalência de transtornos atuais de ansiedade é alta em usuários de substâncias em tratamento em CAPSad. Neste estudo o transtorno de pânico foi o mais prevalente, seguido pelo transtorno de ansiedade generalizada, transtorno obsessivo compulsivo, transtorno de estresse pós-traumático e fobia social. A presença de transtornos de ansiedade foi relacionada a uma maior severidade da dependência sendo cada transtorno específico de ansiedade relacionado a pelo menos uma área da vida do usuário com pior severidade da dependência. As principais áreas com maior severidade da dependência foram as áreas psiquiátrica, uso de álcool e problemas sociofamiliares. A presença de comorbidades de transtornos de ansiedade pode representar uma maior dificuldade no tratamento para o transtorno por uso de substâncias. 


\section{REFERÊNCIAS}

1. AMERICAN PSYCHIATRIC ASSOCIATION (APA). Manual diagnóstico e estatístico de transtornos mentais, 5.ed. (DSM-V). Tradução: Maria Inês Corrêa Nascimento et al. Porto Alegre: Artmed; 2014; 948 p.

2. AMERICAN PSYCHIATRIC ASSOCIATION (APA). Manual diagnóstico e estatístico de transtornos mentais - 4.ed. ver. (DSM-IVTRTM). Tradução de Cláudia Dornelles. Porto Alegre: Artmed; 2008; 880 p.

3. AMORIM P. Mini International Neuropsychiatric Interview (MINI): validação de entrevista breve para diagnóstico de transtornos mentais. Revista Brasileira de Psiquiatria, 2000; 22(3): 106-115.

4. ANDRADE, JDS. Prevalência da comorbidade psiquiátrica em dependentes de drogas de abuso atendidos nos Centros de Atenção Psicossocial álcool e drogas do estado do Sergipe. Dissertação (Mestrado em Ciências Farmacêuticas) Ciências Farmacêuticas. Universidade Federal de Sergipe, São Cristóvão, 2014; 106 p.

5. ANKER JJ, KUSHNER MG. Co-Occurring Alcohol Use Disorder and Anxiety: Bridging Psychiatric, Psychological, and Neurobiological Perspectives. Alcohol Research: Current Reviews, 2019; 40(1): 03.

6. ASSOCIAÇÃO BRASILEIRA DE PSIQUIATRIA (ABP). Abuso e Dependência de Múltiplas Drogas. 2012. Disponível em: https://diretrizes.amb.org.br/_BibliotecaAntiga/abuso e depend\%C3\%AAncia de multiplas drogas.pdf. Acesso em: 05 ago. 2020

7. BRYANT RA, et al. The effect of post-traumatic stress disorder on refugees' parenting and their children's mental health: a cohort study. Lancet Public Health, 2018; 3(5): e249-e258.

8. CAMPÉLO SR, et al. Association between severity of illicit drug dependence and quality of life in psychosocial care center in Brazil: cross sectional study. Health and Quality of Life Outcomes, 2017; 15(1): 223.

9. FORMIGA MB et al. Presence of dual diagnosis between users and non-users of licit and illicit drugs in Brazil. Jornal Brasileiro de Psiquiatria, 2015; 64(4): 288-295.

10. GRANT BF, et al. Prevalence and co-occurrence of substance use disorders and independent mood and anxiety disorders: results from the National Epidemiologic Survey on Alcohol and Related Conditions. Archives of General Psychiatry, 2004; 61 (8): 807-816.

11. IRONS R, SCHNEIDER JP. When is domestic violence a hidden face of addiction? Journal of Psychoactive Drugs, $1997 ; 29(4): 337-$ 344.

12. JAKUSZKOWIAK-WOJTEN K, et al. Impulsivity in anxiety disorders. A critical review. Psychiatria Danubina, 2015; 27(1): 452-455.

13. KESSLER F, et al. Psychometric properties of the sixth version of the Addiction Severity Index (ASI-6) in Brazil. Revista Brasileira de Psiquiatria, 2012; 34(1): 24-33.

14. KESSLER F, et al. Avaliação multidimensional do usuário de drogas e a Escala de Gravidade de Dependência. Revista de Psiquiatria do Rio Grande do Sul, 2010; 32(2): 48-56.

15. KESSLER R, et al. The US National Comorbidity Survey: Overview and future directions. EpidemiologyandPsychiatric Sciences, $1997 ; 6(1): 4-16$

16. KESSLER RC, et al. Lifetime prevalence and age-of-onset distributions of DSM-IV disorders in the National Comorbidity Survey Replication. Archives of General Psychiatry, 2005; 62(6): 593-602.

17. KEYES KM, et al. Anxious and aggressive: the co-occurrence of IED with anxiety disorders. Depression and Anxiety, 2016; 33(2): 101-111.

18. KOOB GF, VOLKOW ND. Neurocircuitry of addiction. Neuropsychopharmacology: official publication of the American College of Neuropsychopharmacology, 2010; 35(1): 217-238.

19. KRUEGER RF. The Structure of Common Mental Disorders. Archives of General Psychiatry, 1999; 56(10): 921-926.

20. KUSHNER MG, et al. The relationship between anxiety disorders and alcohol use disorders: a review of major perspectives and findings. Clinical Psychology Review, 2000; 20(2): 149-171.

21. LAI HM, et al. Prevalence of comorbid substance use, anxiety and mood disorders in epidemiological surveys, 1990-2014: A systematic review and meta-analysis. Drug and Alcohol Dependence, 2015; 154: 1-13.

22. MAGIDSON JF, et al. Comparison of the course of substance use disorders among individuals with and without generalized anxiety disorder in a nationally representative sample. Journal of Psychiatric Research, 2012; 46(5): 659-666.

23. MERIKANGAS KR, et al. Comorbidity of substance use disorders with mood and anxiety disorders: results of the International Consortium in Psychiatric Epidemiology. Addictive Behaviors, 1998; 23(6): 893-907.

24. PAWLUK EJ, KOERNER N. A preliminary investigation of impulsivity in generalized anxiety disorder. Personality and Individual Differences, (2013). 54, 732-737.

25. REGIER DA, et al. Comorbidity of mental disorders with alcohol and other drug abuse: results from the Epidemiologic Catchment Area (ECA) study. JAMA, 1990; 264(19): 2511-18.

26. RITCHIE H, 2019. In: Drug Use. Data source: Global Burden of Disease Collaborative Network. Global Burden of Disease Study 2016 (GBD 2016) Results. Seattle, United States: Institute for Health Metrics and Evaluation (IHME), 2017. Disponível em: https://ourworldindata.org/drug-use. Acesso em: 02 jun. 2020.

27. <https://diretrizes.amb.org.br/ BibliotecaAntiga/abuso e depend\%C3\%AAncia de multiplas drogas. pdf.>Acesso em: 26 jul. 2020.

28. SANDERSON K, ANDREWS G. Prevalence and severity of mental health-related disability and relationship to diagnosis. Psychiatric Services (Washington, D.C.), 2002; 53(1): 80-86.

29. SAREEN J, et al. Anxiety Disorders and Risk for Suicidal Ideation and Suicide Attempts: A Population-Based Longitudinal Study of Adults. Archives of General Psychiatry, 2005; 62(11): 1249-1257.

30. SMITH JP, BOOK SW. Anxiety and substance use disorders: A review. Psychiatric Times, 2008; 25(10): 19-23.

31. SMITH NDL, COTTLER LB. The Epidemiology of Post-Traumatic Stress Disorder and Alcohol Use Disorder. Alcohol Research: Current Review, 2018; 39(2): 113-120.

32. SUMMERFELDT LJ, et al. Impulsivity in obsessive-compulsive disorder: comparisons with other anxiety disorder and within ticrelated subgroups. Personality and Individual Differences, 2004; 36 (3): 539-553.

33. TORRENS M, 2015. Comorbidity of substance use and mental disorders in Europe. Luxembourg: Publications Office of the European Union. Disponível em:<https://www.drugsandalcohol.ie/24863/.>Acesso: 23 may. 2020.

34. TRAN BX, et al. Global research mapping of substance use disorder and treatment 1971-2017: implications for priority setting. Substance Abuse Treatment, Prevention, and Policy, 2019; 14(1): 21

35. VERONA E, et al. Suicide attempts associated with externalizing psychopathology in an epidemiological sample. The American Journal of Psychiatry, 2004; 161(3): 444-451.

36. WORLD HEALTH ORGANIZATION. The World Health Report 2001. Mental Health: New Understanding, New Hope. Geneva: WHO 2001. 178 p. Disponível em:<https://www.who.int/whr/2001/en/index.html.> Acesso em: 2 jun. 2020.

37. ZILBERMAN ML, BLUME SB. Domestic violence, alcohol and substance abuse. Revista. Brasileira de Psiquiatria, 2005; 27(2): 5155. 